\title{
Revisiting geodesic observers in cosmology
}

\author{
Geneviève Vachon $^{\mathrm{a}}$, Robert Vanderwee ${ }^{\mathrm{b}}$, Valerio Faraoni ${ }^{\mathrm{c}}$ (D) \\ Department of Physics and Astronomy, Bishop's University, 2600 College Street, Sherbrooke, QC J1M 1Z7, Canada
}

Received: 6 August 2021 / Accepted: 8 September 2021 / Published online: 16 September 2021

(c) The Author(s) 2021

\begin{abstract}
Geodesic observers in cosmology are revisited. The coordinates based on freely falling observers introduced by Gautreau in de Sitter and Einstein-de Sitter spaces (and, previously, by Gautreau and Hoffmann in Schwarzschild space) are extended to general FLRW universes. We identify situations in which the relation between geodesic and comoving coordinates can be expressed explicitly in terms of elementary functions. In general, geodesic coordinates in cosmology turn out to be rather cumbersome and limited to the region below the apparent horizon.
\end{abstract}

\section{Introduction}

Geodesic observers in radial free fall, and the associated coordinates, were introduced in Schwarzschild spacetime long ago by Ronald Gautreau and Banesh Hoffmann [1] (see also [2-10] and Refs. [11-17] for recent interest). Gautreau used them also in Friedmann-Lemaître-Robertson-Walker (FLRW) cosmology [18,19]. Gautreau's [19] key idea was to use freely falling observers to describe spatially homogeneous and isotropic cosmology, therefore the GautreauHoffmann coordinates in Schwarzschild [1] and their analogue in FLRW $[18,19]$ spacetimes should properly be called "geodesic coordinates". Gautreau's motivation for using these coordinates in cosmology in his 1983 [18] and 1984 [19] papers remains rather obscure, since it is far more natural to describe cosmology from the point of view of comoving observers (those that see the cosmic microwave background spatially homogeneous and isotropic around them, apart from the tiny temperature perturbations $\delta T / T_{0} \simeq 5 \cdot 10^{-5}$ discovered by the COBE satellite in 1992). However, today there is a large literature on the mechanics and thermodynamics of apparent horizons which often require alternative coor-

\footnotetext{
a e-mail: gvachon18@ubishops.ca

b e-mail: rvanderwee20@ubishops.ca

c e-mail: vfaraoni@ubishops.ca (corresponding author)
}

dinates. ${ }^{1}$ Cosmological horizons are increasingly studied as almost trivial examples of apparent horizons to test properties of the analogous (but more complicated) apparent horizons of dynamical black holes. Moreover, cosmology has expanded significantly with (1) the inflationary paradigm of the early universe; (2) the discovery of cosmic microwave background temperature fluctuations in 1992, and (3) the 1998 discovery, made with type Ia supernovae, of the present acceleration of the cosmic expansion. This significant growth of cosmology and of horizon mechanics and thermodynamics motivates the exploration of subjects that were marginal in the past, in particular contemplating alternative coordinate systems in cosmology is more motivated today than it was in the 1980s.

Gautreau [19] restricted himself to spatially flat universes, then further restricted to Einstein-de Sitter universes in which the fluid is a dust and the comoving observers are geodesic [19], or to an empty and locally static de Sitter universe [18] with positive cosmological constant $\Lambda$. Then, he further restricted himself to the discussion of geodesic observers starting their radial free fall from the origin $r=0$. We would like to go beyond all these limitations.

The approach of Refs. $[18,19]$ is rather indirect: Gautreau first writes the FLRW line element as a generic spherically symmetric one using the areal radius as the radial coordinate, and then solves the Einstein equations. Only later, spatial homogeneity and isotropy are imposed. There is no need to do this as the FLRW geometry describing spatial homogeneity and isotropy is well known [23]. Probably due to the lack of a widespread geometric view at the time of writing, ${ }^{2}$ Gautreau's papers $[18,19]$ are rather obscure on several points that can use a transparent geometric clarification or

\footnotetext{
${ }^{1}$ See, for example, Refs. [20,21] for the use of Painlevé-Gullstrand coordinates to describe the thermodynamics of the Schwarzschild horizon and Ref. [22] for the de Sitter horizon.

${ }^{2}$ For example, statements such as "the time coordinate is not suitable for formulating the cosmological principle" and "is tied to one particular galaxy" would lead a reader to suspect that spatial homogeneity and isotropy are coordinate-dependent properties, while spacetime symme-
} 
reformulation. In several other points the reasoning is vague or borderline incorrect (for example, comoving observers are confused with geodesic ones, although this no longer matters when Gautreau specializes to a dust fluid, but becomes crucial when attempting to move beyond this limitation). Certain reasonings are ultimately correct, but this can only be established a posteriori. As a result, the average reader would remain suspicious about the derivation of geodesic coordinates in $[18,19]$ and would avoid using them.

Here we revisit critically the Gautreau construction of geodesic coordinates and we attempt to give a more direct and transparent treatment, while removing the heavy restrictions of Refs. [18, 19]. We begin by using the FLRW geometry in comoving coordinates from the outset, then transforming to Gautreau-Hoffmann-like coordinates employing the areal (or "curvature", or "Schwarzschild-like") radius as the radial coordinate and the proper time of radial geodesic observers as the time coordinate. We elucidate several points not addressed in Refs. [18,19]. As will be clear in the following sections, connecting geodesic coordinates with the more natural comoving coordinates cannot always be done explicitly, in particular for spatially curved FLRW universes. We highlight situations in which the relation between geodesic and comoving time can be calculated explicitly in terms of elementary functions, and we provide explicit examples of physical interest. It turns out that geodesic coordinates in FLRW cosmology are rather cumbersome and only cover the region of FLRW space below the apparent horizon. Indeed, the discussion of radial geodesic observers quickly becomes very involved and, to keep it manageable, we will restrict ourselves to observers initially comoving with the cosmic fluid. Likewise, we only consider FLRW universes sourced by a single perfect fluid in the context of Einstein's theory of gravity.

We follow the notation of Ref. [23]: the metric signature is -+++ and we use units in which Newton's constant $G$ and the speed of light $c$ are unity.

\section{Geodesic and quasi-geodesic observers in FLRW universes}

Here we introduce geodesic coordinates in FLRW spacetime, which are analogous to the Gautreau-Hoffmann coordinates of Schwarzschild spacetime. These new geodesic coordinates in the FLRW universe are associated with observers in radial free fall and starting from rest with respect to the cosmic fluid.

Let us begin by illustrating the difference between geodesic and quasi-geodesic (i.e., comoving) observers in

tries are instead intrinsic and, indeed, comoving coordinates (including comoving time) are adapted to these symmetries.
FLRW space. In general, the worldlines of freely falling observers are (timelike) spacetime geodesics. When the spacetime is sourced by a single perfect fluid, the worldlines of the fluid parcels differ from those of geodesic observers, unless the fluid is a dust [23] because of the pressure gradient $\nabla_{a} P$ acting on them. Specializing to FLRW spacetimes, quasi-geodesics are defined as worldlines which are identical in appearance to a geodesic, yet they differ by the fact that their proper time is a non-affine parameter [24]. A quasigeodesic is the worldline of a particle subject to a pressure gradient parallel to the particle four-velocity (it is clear that, because of spatial isotropy, the four-gradient $\nabla_{c} P$ of the pressure $P(t)$ points in the time direction of comoving observers in a FLRW universe). A quasi-geodesic observer perceives a 3-space which is Lorentz-boosted relative to the proper 3space of a geodesic observer [24]. It was previously shown in [24] that the motion of a fluid particle in a FLRW universe is a radial timelike quasi-geodesic.

The FLRW line element in comoving polar coordinates $(t, r, \vartheta, \varphi)$ is [23]

$d s^{2}=-d t^{2}+a^{2}(t)\left(\frac{d r^{2}}{1-k r^{2}}+r^{2} d \Omega_{(2)}^{2}\right)$,

where $a(t)$ is the scale factor describing the expansion history of the universe, $k$ is the curvature index normalized to $0, \pm 1$, and $d \Omega_{(2)}^{2} \equiv d \vartheta^{2}+\sin ^{2} \vartheta d \varphi^{2}$ is the line element on the unit 2-sphere. We then introduce the areal radius $R(t, r) \equiv$ $a(t) r$, which is analogous to the Schwarzschild radius and is the radial coordinate in the Gautreau coordinate system $[18,19]$. In principle, in a FLRW universe one could also use the proper radius defined by

$R_{p}=a(t) \int \frac{d r}{\sqrt{1-k r^{2}}} \equiv a(t) \chi$,

where $\chi$ is the hyperspherical radius often used in cosmology and

$f(\chi)=r \equiv \begin{cases}\sin \chi & \text { if } k=+1, \\ \chi & \text { if } k=0, \\ \sinh \chi & \text { if } k=-1,\end{cases}$

which turns the FLRW line element (1) into

$d s^{2}=-d t^{2}+a^{2}(t)\left(d \chi^{2}+f^{2}(\chi) d \Omega_{(2)}^{2}\right)$.

$R_{p}$ is a "volume radius" rather than an areal radius and coincides with $R$ only for spatially flat $(k=0)$ universes. Gautreau \& Hoffmann [1] used the areal radius $R$ instead of the proper radius $\int \frac{d R}{\sqrt{1-2 M / R}}$ in the Schwarzschild geometry

$d s^{2}=-\left(1-\frac{2 m}{R}\right) d t^{2}+\frac{d R^{2}}{1-2 m / R}+R^{2} \Omega_{(2)}^{2}$,

hence the analogue of their coordinates in FLRW space should use the areal radius $R$ as well. Defining the proper time 
of geodesic observers and linking it with the time coordinate $t$ of comoving observers is considerably more complicated than introducing the areal radius $R$.

\subsection{Timelike radial geodesics in FLRW}

Let us begin by characterizing the radial timelike geodesics of FLRW spacetime. The non-vanishing Christoffel symbols of the FLRW geometry in comoving coordinates $(t, r, \vartheta, \varphi)$ yield the geodesic equation

$$
\frac{d u^{r}}{d \tau}+2 \frac{\dot{a}}{a} u^{t} u^{r}=0,
$$

where an overdot denotes differentiation with respect to the comoving time $t$. Dividing by $u^{t}=d t / d \tau$, one obtains

$\frac{d}{d t}\left[\ln \left(u^{r}\right)+2 \ln \left(\frac{a}{a_{0}}\right)\right]=0$,

which integrates to

$u^{r}=u_{(0)}^{r} \frac{a_{0}^{2}}{a^{2}}$,

where $u_{(0)}^{r} \equiv u^{r}\left(t_{0}\right)$ is the initial condition at the comoving time $t_{0}$ and $a_{0} \equiv a\left(t_{0}\right)$. The normalization of the fourvelocity $g_{a b} u^{a} u^{b}=-1$ gives

$-\left(u^{t}\right)^{2}+\frac{a^{2}}{1-k r^{2}}\left(u^{r}\right)^{2}=-1$

and Eq. (8) then yields

$u^{t}=\sqrt{1+\frac{\left(u_{(0)}^{r}\right)^{2} a_{0}^{4}}{a^{2}\left(1-k r^{2}\right)}}$,

where the positive sign of the square root is chosen in order for $u^{a}$ to be future-oriented. If the geodesic particle is initially at rest in comoving coordinates (i.e., initially comoving with the cosmic fluid) at time $t_{0}$ and position ${ }^{3} x_{(0)}^{\mu}=$ $\left(t_{0}, r_{0}, \vartheta_{0}, \varphi_{0}\right)$, then the components of its four-velocity are

$u_{(0)}^{\mu}=(1,0,0,0)$,

that is, the four-velocity coincides with that of a radial timelike geodesic. In other words, if the freely falling particle is initially comoving with the cosmic fluid, it remains comoving at all times [24]. This point was missed in Refs. [18, 19]. The time component of the geodesic equation then becomes

$\frac{d u^{t}}{d \tau}+\frac{a \dot{a}}{1-k r^{2}}\left(u_{(0)}^{r}\right)^{2} \frac{a_{0}^{4}}{a^{4}}=0$,

which integrates to

$u^{t}=\frac{d t}{d \tau}=\alpha t+\beta$

\footnotetext{
3 Note that the initial radius of the geodesic observer is not restricted to vanish, as in $[18,19]$.
}

(with $\alpha$ and $\beta$ integration constants) and

$t(\tau)=\frac{\alpha \tau^{2}}{2}+\beta \tau+\gamma$,

where $\gamma$ is another integration constant. For the FLRW cosmic fluid, $t$ is the proper time of the fluid particles, while $\tau$ is the proper time of massive test particles: the two do not coincide unless the fluid is a dust.

\subsection{Pseudo-Painlevé-Gullstrand coordinates}

By switching from comoving radius $r$ to the areal radius $R(t, r) \equiv a(t) r$, and using the relation between differentials $d r=(d R-H R d t) / a$, the FLRW line element (1) assumes the non-diagonal form (dubbed "pseudo-PainlevéGullstrand" form ${ }^{4}$ [28])

$$
\begin{aligned}
d s^{2}= & -\left(1-\frac{H^{2} R^{2}}{1-k R^{2} / a^{2}}\right) d t^{2}-\frac{2 H R}{1-k R^{2} / a^{2}} d t d R \\
& +\frac{d R^{2}}{1-k R^{2} / a^{2}}+R^{2} d \Omega_{(2)}^{2},
\end{aligned}
$$

where $H \equiv \dot{a} / a$ is the (comoving time) Hubble function. In these coordinates, the four-velocity normalization reads

$$
\begin{aligned}
& \left(-1+\frac{H^{2} R^{2}}{1-k R^{2} / a^{2}}\right)\left(u^{t}\right)^{2}-\frac{2 H R}{1-k R^{2} / a^{2}} u^{t} u^{R} \\
& +\frac{\left(u^{R}\right)^{2}}{1-k R^{2} / a^{2}}=-1
\end{aligned}
$$

and can be rewritten in the form

$$
-\left(u^{t}\right)^{2}+\frac{1}{1-k R^{2} / a^{2}}\left(u^{R}-H R u^{t}\right)^{2}=0
$$

that will be useful later. Equation (16) is solved for $u^{R}$, yielding the quadratic equation

$$
\begin{gathered}
\left(u^{R}\right)^{2}-2 H R u^{t} u^{R}-\left(1-H^{2} R^{2}-\frac{k R^{2}}{a^{2}}\right)\left(u^{t}\right)^{2} \\
+1-\frac{k R^{2}}{a^{2}}=0
\end{gathered}
$$

with roots

$u^{R}=H R u^{t} \pm \sqrt{1-\frac{k R^{2}}{a^{2}}} \sqrt{\left(u^{t}\right)^{2}-1}$

We can now relate the components of the four-velocity in pseudo-Painlevé-Gullstrand coordinates to those in comoving coordinates. Since

\footnotetext{
4 This line element resembles the Painlevé-Gullstrand line element for the Schwarzschild geometry but, unless $k=0$, it lacks the defining feature of Painlevé-Gullstrand coordinates that the constant time slices are flat [25-27].
} 
$u^{R} \equiv \frac{d R}{d \tau}=\dot{a} \frac{d t}{d \tau} r+a \frac{d r}{d \tau}=\frac{\dot{a}}{a} R u^{t}+a u^{r}$

and

$u^{r}=\frac{u^{R}}{a}-\frac{H R}{a} u^{t}, \quad u^{R}=H R u^{t}+a u^{r}$,

applying Eqs. (8) and (10) to the second of Eq. (21) gives

$u^{R}=H R \sqrt{1+\frac{\left(u_{(0)}^{r}\right)^{2} a_{0}^{4}}{a^{2}\left(1-k r^{2}\right)}} \pm u_{(0)}^{r} \frac{a_{0}^{2}}{a}$.

We now impose the special initial condition ${ }^{5}$

$$
\begin{aligned}
R\left(t_{0}\right) & =R_{0}, \\
u_{(0)}^{R} & =0,
\end{aligned}
$$

at $t=t_{0}\left(\right.$ or $\left.\tau=\tau_{0}\right)$. Physically, this means that the geodesic clock is released from rest at $R_{0}$, where "at rest" means $d R / d \tau \equiv u^{R}=0$. With these initial conditions, the normalization (16) gives

$$
\left(1-\frac{H_{0}^{2} R_{0}^{2}}{1-k R_{0}^{2} / a_{0}^{2}}\right)\left(u_{(0)}^{t}\right)^{2}=1
$$

and the initial time component

$$
u_{(0)}^{t}=\sqrt{\frac{1-k R_{0}^{2} / a_{0}^{2}}{1-H_{0}^{2} R_{0}^{2}-k R_{0}^{2} / a_{0}^{2}}} .
$$

Substituting this expression into the first of Eq. (21) yields

$u_{(0)}^{r}=-\frac{H_{0} R_{0} u_{(0)}^{t}}{a_{0}}$

and, finally,

$u_{(0)}^{r}=-\frac{H_{0} R_{0}}{a_{0}} \sqrt{\frac{1-k R_{0}^{2} / a_{0}^{2}}{1-H_{0}^{2} R_{0}^{2}-k R_{0}^{2} / a_{0}^{2}}}$.

Equation (28) agrees with what one obtains by setting $u_{0}^{R}=0$ in Eqs. (22) and (17). Using the normalisation of the fourvelocity in comoving coordinates and Eq. (28), one obtains

$u^{t}=\sqrt{\frac{a_{0}^{2} H_{0}^{2} R_{0}^{2}}{a^{2}\left(1-k R^{2} / a^{2}\right)} \frac{1-k R_{0}^{2} / a_{0}^{2}}{1-k R_{0}^{2} / a_{0}^{2}-H_{0}^{2} R_{0}^{2}}+1}$

along the radial timelike geodesics with the special initial condition (23), (24). As a check, Eq. (29) agrees with Eq. (26) at the spacetime point $\left(t_{0}, R_{0}, \vartheta_{0}, \varphi_{0}\right)$.

\footnotetext{
${ }^{5}$ Gautreau imposes the special initial position $R_{0}=0$ invoking the cosmological principle - the meaning of this statement is unclear. We do not impose this unnecessary restriction and the geodesic clock can be dropped from any initial position below the apparent horizon.
}

The use of Eqs. (22) and (28) then leads to

$$
u^{R}=H R \sqrt{1+\frac{a_{0}^{4}\left(u_{(0)}^{r}\right)^{2}}{a^{2}\left(1--k r^{2}\right)}} \pm u_{(0)}^{r} \frac{a_{0}^{2}}{a^{2}}
$$

$$
\begin{aligned}
&= H R \sqrt{1+\frac{a_{0}^{2} H_{0}^{2} R_{0}^{2}}{a^{2}\left(1-k r^{2}\right)} \frac{1-k R_{0}^{2} / a_{0}^{2}}{1-H_{0}^{2} R_{0}^{2}-k R_{0}^{2} / a_{0}^{2}}} \\
& \mp \frac{H_{0} R_{0} a_{0}}{a} \sqrt{\frac{1-k R_{0}^{2} / a_{0}^{2}}{1-k R_{0}^{2} / a_{0}^{2}-H_{0}^{2} R_{0}^{2}}},
\end{aligned}
$$

which (as a check) satisfies $u_{(0)}^{R}=0$ at $R_{0}$. For an observer initially at rest (i.e., $u_{(0)}^{R}=0$ ), the relation (21) suggests that

$u_{(0)}^{r}=-\frac{H_{0} R_{0} u_{(0)}^{t}}{a_{0}}<0:$

of course, if this geodesic observer is at rest in the GautreauHoffmann sense, it is left behind by the comoving observers and its radial velocity according to the comoving observers is negative.

In the following we need the components of the fourvelocity covector

$$
\begin{aligned}
u_{t}= & g_{t \alpha} u^{\alpha} \\
= & \frac{a_{0} H_{0} R_{0} H R}{a\left(1-k R^{2} / a^{2}\right)} \sqrt{\frac{1-k R_{0}^{2} / a_{0}^{2}}{1-k R_{0}^{2} / a_{0}^{2}-H_{0}^{2} R_{0}^{2}}} \\
& -\sqrt{1+\frac{a_{0}^{2} H_{0}^{2} R_{0}^{2}}{a^{2}\left(1-k R^{2} / a^{2}\right)} \frac{1-k R_{0}^{2} / a_{0}^{2}}{1-k R_{0}^{2} / a_{0}^{2}-H_{0}^{2} R_{0}^{2}}}
\end{aligned}
$$

and

$$
\begin{aligned}
u_{R} & =g_{R \alpha} u^{\alpha}, \\
& =\mp \frac{a_{0} H_{0} R_{0}}{a\left(1-k R^{2} / a^{2}\right)} \sqrt{\frac{1-k R_{0}^{2} / a_{0}^{2}}{1-k R_{0}^{2} / a_{0}^{2}-H_{0}^{2} R_{0}^{2}}} .
\end{aligned}
$$

\subsection{Geodesic coordinates}

The Gautreau-Hoffmann-like geodesic coordinates are ( $\tau$, $R, \vartheta, \varphi)$, where $\tau$ is the proper time of clocks freely falling from rest (i.e., $u_{(0)}^{R}=0$ initially). The relation between $\tau$ and the comoving time $t$ is given by $u^{t} \equiv d t / d \tau$ and $d \tau=d t / u^{t}$. 
In finite terms,

$$
\begin{aligned}
\tau= & \int \frac{d t}{u^{t}} \\
= & \int d t\left[\frac{a_{0}^{2} H_{0}^{2} R_{0}^{2}}{a^{2}\left(1-k R^{2} / a^{2}\right)}\right. \\
& \left.\times \frac{1-k R_{0}^{2} / a_{0}^{2}}{1-k R_{0}^{2} / a_{0}^{2}-H_{0}^{2} R_{0}^{2}}+1\right]^{-1 / 2}
\end{aligned}
$$

or

$$
\begin{aligned}
\tau= & \int d t \sqrt{\left(a^{2}-k R^{2}\right)\left(1-\frac{k R_{0}^{2}}{a_{0}^{2}}-H_{0}^{2} R_{0}^{2}\right)} \\
& \times\left[\left(a^{2}-k R^{2}\right)\left(1-\frac{k R_{0}^{2}}{a_{0}^{2}}-H_{0}^{2} R_{0}^{2}\right)\right. \\
& \left.+a_{0}^{2} H_{0}^{2} R_{0}^{2}\left(1-\frac{k R_{0}^{2}}{a_{0}^{2}}\right)\right]^{-1 / 2} .
\end{aligned}
$$

Using the notation

$\alpha_{0} \equiv 1-\frac{k R_{0}^{2}}{a_{0}^{2}}-H_{0}^{2} R_{0}^{2}$,

$\beta_{0} \equiv H_{0}^{2} R_{0}^{2}\left(a_{0}^{2}-k R_{0}^{2}\right)$

the $\tau$-coordinate is expressed by the integral

$\tau=\sqrt{\alpha_{0}} \int d t \sqrt{\frac{a^{2}-k R^{2}}{\left(a^{2}-k R^{2}\right) \alpha_{0}+\beta_{0}}}$,

where $^{6} a=a(t)$.

\section{Spatially flat FLRW universes}

Motivated by modern cosmological observations, let us restrict to a spatially flat FLRW universe. For $k=0$, the Gautreau-Hoffmann-like geodesic time reduces to

$\tau=\sqrt{\alpha_{0}} \int d t \sqrt{\frac{a^{2}}{\alpha_{0} a^{2}+\beta_{0}}}$,

and it is sometimes possible to express it in terms of elementary functions. Below, we discuss these integrability situations.

\subsection{Power-law scale factor}

Let us consider first a power-law scale factor, which always occurs for a spatially flat FLRW universe dominated by a

\footnotetext{
${ }^{6}$ Switching to conformal time does not help in computing this integral.
}

single perfect fluid with constant barotropic equation of state $P=w \rho[23]$,

$a(t)=a_{*} t^{p}$,

where $a_{*}$ is a constant. In this case, it is

$\tau=\sqrt{\alpha_{0}} a_{*} \int d t t^{p}\left(\alpha_{0} a_{*}^{2} t^{2 p}+\beta_{0}\right)^{-1 / 2}$.

According to the Chebyshev theorem of integration [29,30], the integral

$\int d t t^{p}\left(A+B t^{r}\right)^{q}$

where $A, B, p, q, r$ are constants and $r \neq 0, p, q, r \in \mathbb{Q}$, is expressed in terms of a finite number of elementary functions if and only if at least one of

$\frac{p+1}{r}, \quad q, \quad \frac{p+1}{r}+q$

is an integer [29,30].

An alternative approach consists of using a representation of the integral (43) in terms of a hypergeometric series and noting that the assumptions of the Chebyshev theorem are equivalent to the condition for this series to reduce to a finite sum (this equivalent condition was noted several times in the context of two-fluid cosmologies, for which the Friedmann equation reduces to an integral of the same type [31-35]).

In our case we can assume $p \in \mathbb{Q}$. In general, if the equation of state of the cosmic fluid has the barotropic form $P=w \rho$ with $w=$ const., then

$a(t)=a_{*} t^{\frac{2}{3(w+1)}}$,

and $w \in \mathbb{Q}$ implies that $p=2 /[3(w+1)] \in \mathbb{Q}$. Most values of the equation of state parameter $w$ used in the cosmological literature are indeed rational but, if this is not the case, one can always approximate $w \in \mathbb{R}$ with its rational approximation, still satisfying the cosmological observations to the required precision. We have then that $p, r=2 p, q=-1 / 2 \in \mathbb{Q}$ and

$$
\frac{p+1}{r}=\frac{p+1}{2 p}, \quad q=-\frac{1}{2} \notin \mathbb{Z}, \quad \frac{p+1}{r}+q=\frac{1}{2 p} .
$$

We have

$$
\frac{p+1}{r}=m \in \mathbb{Z}
$$

if and only if $p=1 /(2 m-1)$, while

$$
\frac{p+1}{r}+q=m \in \mathbb{Z}
$$

if and only if $p=1 / 2 m$, so at least one of

$$
\frac{p+1}{r}, q, \frac{p+1}{r}+q \in \mathbb{Z}
$$


Table 1 The equation of state parameter for a few values of $n$

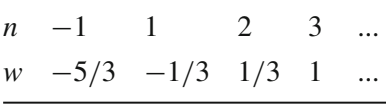

if $p=1 / n$, where $n= \pm 1, \pm 2, \pm 3, \ldots$ This list includes several well known equations of state in cosmology. Setting

$p=\frac{2}{3(w+1)}, \quad w_{n}=\frac{2 n-3}{3}$,

we have the equations of state listed in Table 1.

Let us discuss, as examples, two of these integrability cases, plus one not given by the Chebyshev theorem.

\subsection{Radiation fluid}

The equation of state and power-law scale factor associated with a $k=0$ FLRW universe filled with radiation are $P=$ $\rho / 3$ and $a(t)=a_{*} \sqrt{t}$, which give

$$
\begin{aligned}
\tau= & \sqrt{\alpha_{0}} a_{*} \int d t \sqrt{t}\left(\alpha_{0} a_{*}^{2} t+\beta_{0}\right)^{-1 / 2} \\
= & \frac{1}{\alpha_{0} a_{*}^{2}}\left[\sqrt{\alpha_{0} a_{*}^{2} t+\beta_{0}} \sqrt{\alpha_{0} a_{*}^{2} t}\right. \\
& \left.-\beta_{0} \sinh ^{-1}\left(\sqrt{\frac{\alpha_{0} a_{*}^{2} t}{\beta_{0}}}\right)\right] .
\end{aligned}
$$

\subsection{Stiff fluid/free scalar field}

A universe filled with a stiff fluid with equation of state ${ }^{7}$ $P=\rho$ has scale factor $a(t)=a_{*} t^{1 / 3}$, yielding the time coordinate

$$
\begin{aligned}
\tau & =\sqrt{\alpha_{0}} a_{*} \int d t t^{1 / 3}\left[\alpha_{0} a_{*}^{2} t^{2 / 3}+\beta_{0}\right]^{-1 / 2} \\
& =\frac{1}{\alpha_{0}^{3 / 2} a_{*}^{3}}\left(\alpha_{0} a_{*}^{2} t^{2 / 3}+2 \beta_{0}\right) \sqrt{\alpha_{0} a_{*}^{2} t^{2 / 3}+\beta_{0}} .
\end{aligned}
$$

\section{4 de Sitter space}

The de Sitter universe with scale factor $a(t)=a_{*} \mathrm{e}^{H t}$, $H=$ const. is another special case in which the GautreauHoffmann-like geodesic time can be computed explicitly, giving

$$
\begin{aligned}
\tau & =\sqrt{\alpha_{0}} a_{*} \int d t \mathrm{e}^{H t}\left(\alpha_{0} a_{*}^{2} \mathrm{e}^{2 H t}+\beta_{0}\right)^{-1 / 2} \\
& =\frac{1}{H} \tanh ^{-1}\left(\frac{\mathrm{e}^{H t}}{\sqrt{\mathrm{e}^{2 H t}+\frac{\beta_{0}}{\alpha_{0} a_{*}^{2}}}}\right) .
\end{aligned}
$$

\footnotetext{
$\overline{7}$ It is well known that a stiff fluid is equivalent to a free scalar field.
}

The relation $\tau=\tau(t)$ can be inverted to find $t(\tau)$ and $a(\tau)$, obtaining

$t=\frac{1}{H} \ln \left[\sqrt{\frac{\beta_{0}}{\alpha_{0} a_{*}^{2}}} \sinh (H \tau)\right]$.

The scale factor as a function of $\tau$ is then

$a(\tau)=\sqrt{\frac{\beta_{0}}{\alpha_{0}}} \sinh (H \tau)$.

3.5 Range of validity of the geodesic coordinates

Let us establish the range of validity of the GautreauHoffmann-like geodesic coordinate patch. First, remember that the components of the four-velocity of radial geodesic observers are

$u^{t}=\sqrt{\frac{\beta_{0}}{\alpha_{0} a^{2}\left(1-k R^{2} / a^{2}\right)}+1}$,

$u^{R}=H R \sqrt{\frac{\beta_{0}}{\alpha_{0} a^{2}\left(1-k R^{2} / a^{2}\right)}+1}-\frac{1}{a} \sqrt{\frac{\beta_{0}}{\alpha_{0}}}$,

with $\alpha_{0}$ and $\beta_{0}$ given by Eqs. (37) and (38). For a flat FLRW universe it is $1-H_{0}^{2} R_{0}^{2}>0$ and $a_{0} H_{0} R_{0} \geq 0$ for $R_{0}<$ $H_{0}^{-1}$, then the time component of the four-velocity is

$u^{t}=\sqrt{\frac{\beta_{0}}{\alpha_{0} a^{2}}+1}$,

which is defined only for $\beta_{0} /\left(\alpha_{0} a^{2}\right)>-1$. The radial component $u^{R}$ of the four-velocity of a radial geodesic observer is defined only if $\alpha_{0}>0$, and one concludes that the geodesic coordinates must satisfy $R_{0}<H_{0}^{-1}$.

For a curved $(k= \pm 1)$ FLRW universe, $\alpha_{0}$ must be positive again, which is equivalent to the constraint

$R_{0}<\frac{1}{\sqrt{H_{0}^{2}+k / a_{0}^{2}}}$,

where the right hand side is the radius of the apparent cosmological horizon [28]. If the universe is negatively curved, then

$\beta_{0}=H_{0}^{2} R_{0}^{2}\left(a_{0}^{2}+R_{0}^{2}\right)>0$

while, if it is positively curved,

$\beta_{0}=H_{0}^{2} R_{0}^{2}\left(a_{0}^{2}-R_{0}^{2}\right) \geq 0$

for $R_{0} \leq a_{0}$, which is always satisfied due to the constraint on $u^{t}$. The latter gives

$\frac{\beta_{0}}{\alpha_{0} a^{2}\left(1-k R^{2} / a^{2}\right)}>0$. 


\subsection{Line element in geodesic coordinates}

Let us attempt to express the FLRW line element in Gautreau-Hoffmann-like geodesic coordinates. Eq. (34) can be used again to express $d t$ in terms of $d \tau$,

$d t=u^{t} d \tau=\sqrt{\frac{\beta_{0}}{\alpha_{0} a^{2}\left(1-k R^{2} / a^{2}\right)}+1} d \tau$

which, substituted in the FLRW line element in pseudoPainlevé-Gullstrand coordinates (15) produces the nonvanishing metric components

$g_{\tau \tau}=-\left(1-\frac{H^{2} R^{2}}{1-k R^{2} / a^{2}}\right)\left(\frac{\beta_{0}}{\alpha_{0} a^{2}\left(1-k R^{2} / a^{2}\right)}+1\right)$,

$g_{\tau R}=-\frac{H R}{1-k R^{2} / a^{2}} \sqrt{\frac{\beta_{0}}{\alpha_{0} a^{2}\left(1-k R^{2} / a^{2}\right)}+1}$,

$g_{R R}=\frac{1}{1-k R^{2} / a^{2}}$,

$g_{\vartheta \vartheta}=R^{2}$,

$g_{\varphi \varphi}=R^{2} \sin ^{2} \vartheta$,

in geodesic coordinates, where now $a=a(t(\tau))$ and $H=$ $H(t(\tau))$. When $d \tau=0$, the Riemannian 3-spaces are the same as in the comoving FLRW foliation.

Let us consider again the special case of the de Sitter universe. Its line element in geodesic coordinates can be diagonalized by introducing a new radial coordinate $\rho=\rho(\tau, R)$ by

$d \rho=\frac{1}{F}(\beta d \tau+d R)$,

where $\beta(\tau, R)$ must be determined a posteriori so that the cross-term $d \tau d \rho$ disappears, while $F(\tau, R)$ is an integrating factor satisfying

$\frac{\partial}{\partial R}\left(\frac{1}{F}\right)=\frac{\partial}{\partial \tau}\left(\frac{\beta}{F}\right)$

in order to guarantee that $d \tau$ is an exact differential. Using $d R=F d \rho-\beta d \tau$, one obtains

$$
\begin{aligned}
d s^{2}= & -\left[\left(1-H^{2} R^{2}\right) \operatorname{coth}^{2}(H \tau)\right. \\
& \left.-\beta^{2}-2 H R \operatorname{coth}(H \tau) \beta\right] d \tau^{2} \\
& -2 F[H R \operatorname{coth}(H \tau)+\beta] d \rho d \tau \\
& +F^{2} d \rho^{2}+R^{2} d \Omega_{(2)}^{2} .
\end{aligned}
$$

The choice

$\beta=-H R \operatorname{coth}(H \tau)$ eliminates the time-radius cross-term and diagonalizes the line element, that becomes

$d s^{2}=-\operatorname{coth}^{2}(H \tau) d \tau^{2}+F^{2} d \rho^{2}+R^{2}(\tau, \rho) d \Omega_{(2)}^{2}$.

With the choice (76) of $\beta$, the general solution of Eq. (72) is

$F(\tau, R)=\frac{A \exp \left[-(\lambda / 2) R^{2}\right]}{\tanh (H \tau)}[\cosh (H \tau)]^{\lambda / H^{2}}$,

where $A$ is an integration constant and $\lambda$ is a separation constant (see Appendix A). Setting $A=1$ and $\lambda=0$ so that

$F(\tau, R)=\operatorname{coth}(H \tau)$

produces the diagonal de Sitter line element

$d s^{2}=\operatorname{coth}^{2}(H \tau)\left(d \rho^{2}-d \tau^{2}\right)+R^{2}(\tau, \rho) d \Omega_{(2)}^{2}$.

\section{Concluding remarks}

We have revisited geodesic and quasi-geodesic observers in FLRW universes, removing the restrictions intrinsic in Gautreau's previous work, which was limited to de Sitter and Einstein-de Sitter universes $[18,19]$. In general, geodesic coordinates turn out to be rather cumbersome in generic FLRW spaces, especially those with curved spatial sections. In particular, one would like to express the geodesic time $\tau$ as a function of the comoving time $t$, the parameters, and the initial conditions along the radial timelike geodesics of FLRW space. The time measured by freely falling clocks (i.e., the proper time of radial geodesic massive observers) is expressed by an integral that, in general, cannot be computed explicitly in terms of elementary functions, even in spatially flat FLRW universes. This situation, however, improves in most situations of practical interest, including the case of a power-law scale factor and, of course, in de Sitter space. We have provided explicit solutions for a radiation fluid, a stiff fluid, and empty de Sitter space. The latter, being locally static, is rather similar to the Schwarschild geometry and was already discussed by Gautreau [18], who had already used geodesic coordinates in Schwarzschild space in his earlier joint paper with Hoffmann [1]. For power-law scale factors $a(t)$ in $k=0$ FLRW universes, we have identified all the situations in which the geodesic time $\tau$ can be expressed explicitly in terms of comoving time by making use of the Chebyshev theorem of integration [29,30], under the mild assumption that the equation of state parameter $w$ is a rational number. Alternatively, one can use the representation of the integral (43) in terms of a hypergeometric function and note that the assumptions of the Chebyshev theorem leading to integrability are equivalent to the conditions for the truncation of the hypergeometric series to a finite sum (this 
mathematical condition was noted several times in the different context of two-fluid cosmologies [31-35]).

The range of validity of geodesic coordinates is also limited: the radial coordinate is restricted to the region below the apparent horizon of the FLRW universe and, therefore, it is not expected that geodesic coordinates will be useful for the thermodynamics of this apparent horizon since they cannot penetrate it (contrary to the Kruskal-Szekeres coordinates [36,37], the Painlevé-Gullstrand coordinates $[25,26]$, or their Martel-Poisson generalization [27] in the Schwarzschild geometry). In this region below the apparent horizon, the geodesic coordinates describe the internal clock of dark matter or of free-falling test particles.

Acknowledgements This work is supported by the Natural Sciences \& Engineering Research Council of Canada (Grant no. 2016-03803 to V.F.) and by Bishop's University.

Data Availability Statement This manuscript has no associated data or the data will not be deposited. [Authors' comment: Due to the theoretical nature of this work, there are no data involved.].

Open Access This article is licensed under a Creative Commons Attribution 4.0 International License, which permits use, sharing, adaptation, distribution and reproduction in any medium or format, as long as you give appropriate credit to the original author(s) and the source, provide a link to the Creative Commons licence, and indicate if changes were made. The images or other third party material in this article are included in the article's Creative Commons licence, unless indicated otherwise in a credit line to the material. If material is not included in the article's Creative Commons licence and your intended use is not permitted by statutory regulation or exceeds the permitted use, you will need to obtain permission directly from the copyright holder. To view a copy of this licence, visit http://creativecomm ons.org/licenses/by/4.0/.

Funded by SCOAP ${ }^{3}$.

\section{Appendix A: Integrating factor for the de Sitter universe}

Using the variable $u(\tau, R) \equiv 1 / F$, we have

$\frac{\partial u}{\partial R}=\frac{\partial}{\partial \tau}[-H R u \operatorname{coth}(H \tau)]$;

assume the ansatz

$u(\tau, R)=T(\tau) S(R)$,

then it is

$T \frac{d S}{d R}=-R S \frac{d}{d \tau}[H \operatorname{coth}(H \tau) T]$.

Dividing both sides by $R S T$ gives

$\frac{1}{R S} \frac{d S}{d R}=-\frac{1}{T} \frac{d}{d \tau}[H \operatorname{coth}(H \tau) T] ;$

the left-hand side depends only on $R$ while the right-side depends only on $\tau$, hence it must be

$\frac{1}{R S} \frac{d S}{d R}=\lambda=-\frac{1}{T} \frac{d}{d \tau}[H \operatorname{coth}(H \tau) T]$, where $\lambda$ is a separation constant. The function $S(R)$ obeys

$\frac{1}{S} \frac{d S}{d R}=\lambda R$,

which integrates to

$S=A_{1} \exp \left(\frac{\lambda R^{2}}{2}\right)$

with $A_{1}$ an integration constant. The time part $T(\tau)$ satisfies the equation

$\frac{d}{d \tau}[H \operatorname{coth}(H \tau) T]=-\lambda T$,

which yields

$H \operatorname{coth}(H \tau) \frac{d T}{d \tau}=-\left(\lambda+\frac{d}{d \tau}[H \operatorname{coth}(H \tau)]\right) T$

and

$T=\frac{A_{2}}{H} \tanh (H \tau)[\cosh (H \tau)]^{-\lambda / H^{2}}$.

The general solution for $u(\tau, R)$ is, therefore,

$u(\tau, R)=\frac{A_{1} A_{2}}{H} \exp \left(\frac{\lambda R^{2}}{2}\right) \tanh (H \tau)[\cosh (H \tau)]^{-\lambda / H^{2}}$.

Then, $F(\tau, R)$ is of the form

$F(\tau, R)=\frac{A \exp \left[-(\lambda / 2) R^{2}\right]}{\tanh (H \tau)}[\cosh (H \tau)]^{\lambda / H^{2}}$,

where $A$ is an integration constant.

\section{References}

1. R. Gautreau, B. Hoffmann, The Schwarzschild radial coordinate as a measure of proper distance. Phys. Rev. D 17, 2552-2555 (1978). https://doi.org/10.1103/PhysRevD.17.2552

2. C. Ftaclas, J.M. Cohen, Generalized radial observers and the Reissner-Nordström field. Phys. Rev. D 21, 2103 (1980)

3. R. Gautreau, Imbedding a Schwarzschild mass into cosmology. Phys. Rev. D 29(2), 198 (1984). https://doi.org/10.1103/ PhysRevD.29.198

4. R. Gautreau, Light cones inside the Schwarzschild radius. Am. J. Phys. 63, 431 (1995)

5. R. Gautreau, Newton's absolute time and space in general relativity. Am. J. Phys. 68, 350 (2000)

6. M.R. Francis, A. Kosowsky, Geodesics in the generalized Schwarzschild solution. Am. J. Phys. 72, 1204-1209 (2004). https://doi.org/10.1119/1.1701846. arXiv:gr-qc/0311038

7. A.B. Whiting, The expansion of space: free particle motion and the cosmological redshift. Observatory 124, 174 (2004). arXiv:astro-ph/0404095

8. T.K. Finch, Coordinate families for the Schwarzschild geometry based on radial timelike geodesics. Gen. Relativ. Gravit. 47(5), 56 (2015). https://doi.org/10.1007/s10714-015-1891-7. arXiv:1211.4337 [gr-qc] 
9. A. Mitra, Friedmann-Robertson-Walker metric in curvature coordinates and its applications. Gravit. Cosmol. 19, 134-137 (2013). https://doi.org/10.1134/S0202289313020072

10. C. Posada, Imbedding a Reissner-Nordström charged mass into cosmology. arXiv:1405.6697 [gr-qc]

11. C. MacLaurin, Schwarzschild spacetime under generalised Gullstrand-Painlevé slicing. https://doi.org/10.1007/ 978-3-030-18061-4_9. arXiv:1911.05988 [gr-qc]

12. C. MacLaurin, Clarifying spatial distance measurement. arXiv: 1911.07500 [gr-qc]

13. C. MacLaurin, Expanding space, redshifts, and rigidity: conceptual issues in cosmology. arXiv:1911.05415 [gr-qc]

14. M. Kerachian, Uniformly accelerated traveler in an FLRW universe. Phys. Rev. D 101(8), 083536 (2020). https://doi.org/10. 1103/PhysRevD.101.083536. arXiv:1911.05436 [gr-qc]

15. V. Faraoni, F. Atieh, Turning a Newtonian analogy for FLRW cosmology into a relativistic problem. Phys. Rev. D 102(4), 044020 (2020). https://doi.org/10.1103/PhysRevD.102.044020. arXiv:2006.07418 [gr-qc]

16. J.P.S. Lemos, D.L.F.G. Silva, Maximal extension of the Schwarzschild metric: from Painlevé-Gullstrand to KruskalSzekeres. Ann. Phys. 430, 168497 (2021). https://doi.org/10.1016/ j.aop.2021.168497. arXiv:2005.14211 [gr-qc]

17. A.A. Grib, Y.V. Pavlov, Some effects of different coordinate systems in cosmology. Eur. Phys. J. Plus 136(3), 318 (2021). https://doi.org/10.1140/epjp/s13360-021-01249-7. arXiv:2012.14110 [gr-qc]

18. R. Gautreau, Geodesic coordinates in the de Sitter universe. Phys. Rev. D 27, 764 (1983)

19. R. Gautreau, Curvature coordinates in cosmology. Phys. Rev. D 29, 186 (1984)

20. M.K. Parikh, F. Wilczek, Hawking radiation as tunneling. Phys. Rev. Lett. 85, 5042-5045 (2000). https://doi.org/10.1103/ PhysRevLett.85.5042. arXiv:hep-th/9907001

21. S.T. Hong, Y.W. Kim, Y.J. Park, GEMS embeddings of Schwarzschild and RN black holes in Painlevé-Gullstrand spacetimes. arXiv:2011.08351 [gr-qc]

22. M.K. Parikh, New coordinates for de Sitter space and de Sitter radiation. Phys. Lett. B 546, 189-195 (2002). https://doi.org/10. 1016/S0370-2693(02)02701-6. arXiv:hep-th/0204107

23. R.M. Wald, General Relativity (Chicago University Press, Chicago, 1984)

24. V. Faraoni, G. Vachon, When Painlevé-Gullstrand coordinates fail. Eur. Phys. J. C 80(8), 771 (2020). https://doi.org/10.1140/epjc/ s10052-020-8345-4. arXiv:2006.10827 [gr-qc]

25. P. Painlevé, a mécanique classique et la théorie de la relativité. Compt. Rendu Acad. Sci. (Paris) 173, 677 (1921)
26. A. Gullstrand, Allgemeine Lösung des statischen Einkörperproblems in der Einsteinschen Gravitationstheorie. Arkiv. Mat. Astron. Fys. 16, 1 (1912)

27. K. Martel, E. Poisson, Regular coordinate systems for Schwarzschild and other spherical space-times. Am. J. Phys. 69, 476-480 (2001). https://doi.org/10.1119/1.1336836. arXiv:gr-qc/0001069

28. V. Faraoni, Cosmological and Black Hole Apparent Horizons. Lecture Notes Physics, vol. 907 (Springer, New York, 2015), pp. 1-199. (10.1007/978-3-319-19240-6)

29. P.L. Chebyshev, Sur l'integration des différentielles irrationnelles. J. Math. (Ser. 1) 18, 87-111 (1853)

30. E.A. Marchisotto, G.-A.Zakeri, An invitation to integration in finite terms. Coll. Math. J. 25, 295-308 (1994)

31. K.C. Jacobs, Spatially homogeneous and Euclidean cosmological models with shear. Astrophys. J. 153, 661 (1968)

32. C.B.G. McIntosh, I. Robertson-Walker metric. Aust. J. Phys. 25, 75-82 (1972)

33. C.B.G. McIntosh, J.M. Foyster, Cosmological models with two fluids II. Conformal and conformally flat metrics. Aust. J. Phys. 25, 83-89 (1972)

34. S. Chen, G.W. Gibbons, Y. Li, Y. Yang, Friedmann's equations in all dimensions and Chebyshev's theorem. JCAP 12, 035 (2014). https://doi.org/10.1088/1475-7516/2014/12/035. arXiv:1409.3352 [astro-ph.CO]

35. V. Faraoni, S. Jose, S. Dussault, Multi-fluid cosmology in Einstein gravity: analytical solutions. arXiv:2107.12488 [gr-qc]

36. M.D. Kruskal, Maximal extension of Schwarzschild metric. Phys. Rev. 119, 1743 (1960)

37. G. Szekeres, On the singularities of a Riemannian manifold. Publ. Mat. Debr. 7, 285 (1960) 